\title{
802.11 Mesh Networks with Two-Radio Access Points
}

\author{
Jing Zhu \\ jing.z.zhueintel.com \\ Communications Technology Lab \\ Intel Corporation, 2111 NE 25th Ave., JF3-206 \\ Hillsboro, OR 97124 USA
}

\author{
Sumit Roy \\ roydee.washington.edu \\ Dept. of Electrical Engineering \\ University of Washington, Box 352500 \\ Seattle, WA 98195-2500 U.S.A.
}

\begin{abstract}
In this future-looking work, we propose a mesh (multi-hop) architecture based on two-radio .11 Access Points (AP) and examine achievable aggregate throughput by exploiting spatial reuse and multiple channels. A suitable distributed clustering is used to self-organize the network for channel allocation; all communications between nodes in the same cluster (intra-cluster) use the secondary radio and a common channel selected based on an algorithm that minimizes the co-channel interference (CCI). This dramatically reduces complexity compared to per-packet channel switching approaches. All intercluster communications are performed on a common channel using the default (primary) radio. Backward compatibility is guaranteed by allowing legacy single-channel APs to connect to the new two-radio devices through the common default radio. Simulation results for large-scale $802.11 b$ networks demonstrate the significant improvement in one-hop aggregate throughput. Specifically, the new two-radio multi-channel mesh solution more than doubles the aggregate throughput compared to the traditional single-radio single-channel mesh.
\end{abstract}

\section{INTRODUCTION}

802.11 wireless LANs for broadband wireless access constitute a growing success story. Currently, their deployment in single-cell (i.e. single AP) scenarios (homes, small business and isolated hotspots) is well-supported by current .11 technology. However, their performance in multi-cell deployments (large enterprizes, public hotspots and dense clusters of homes/apartments) where each cell is served by its own access point (AP) is much less understood or supported with current technology. Of the several issues, the primary one is of scalability - currently, all APs are directly connected (typically via Ethernet) to an Internet gateway. This (wired connection per AP) dramatically increases the cost of deploying a large scale WLAN network.

A possible solution to this problem is connecting APs wirelessly to form a (static) multi-hop .11 (mesh) network (see Fig.1). Interest in such an approach is indicated not only by the newly formed Mesh Task Group within IEEE 802.11 but also mesh solutions offered by several companies [1], [2], [3] to list a few. Such a future wireless AP-AP mesh network requires both protocol and architectural extensions to current .11 networks for which there does not exist any standardized inter-AP connectivity protocol. Assuming the existence of such inter-AP connectivity allows enhanced aggregate network throughput via exploitation of co-channel spatial reuse, i.e. multiple concurrent transmissions on the same channel may be allowed if the co-channel transmission are sufficiently separated. This naturally suggests a structured cellular layout; however due to the ad-hoc nature of AP deployment in most cases, some form of self-organization based on clustering is desirable to achieve increased aggregate throughput. The clustering should be achieved in a distributed manner; once the network clusters are formed, a suitable channel is determined for each cluster based on minimizing the aggregate interference. As we demonstrate via simulations in this work, the aggregate network throughput of such a inter-AP mesh is governed by the following key network parameters:

$\mathrm{K}$ : number of concurrent active links per channel (degree of co-channel spatial reuse);

W: max. data rate per channel;

$\mathrm{N}$ : number of orthogonal channels available $(\mathrm{N}=8$ in 802.11 a, and $\mathrm{N}=3$ in $802.11 \mathrm{~b}$ )

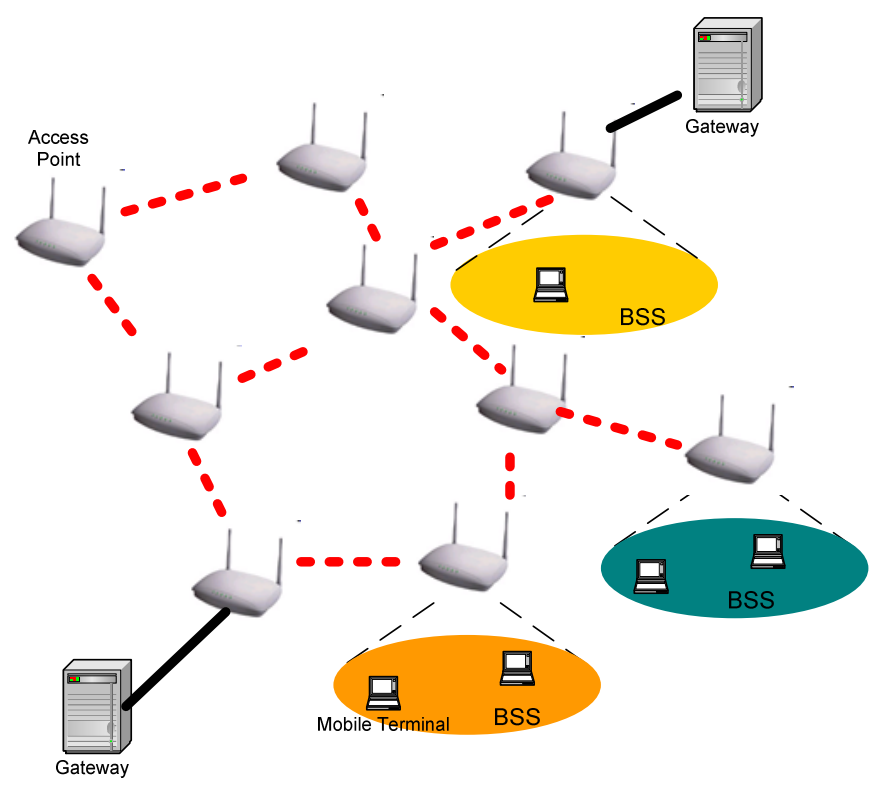

Fig. 1. Wireless AP-to-AP mesh Networks

Current .11 APs are all single-radio which can however be set to any of the available multiple orthogonal channels. Selecting an optimal channel-to-link assignment is potentially complicated with a single radio, and hence its benefits 
(increased aggregate throughput by exploiting the available multiple channels) has not been realized in practice. Our proposal: a two-radio multi-channel mesh where each node is equipped with two similar radio interfaces for APAP communication ${ }^{1}$, can effectively exploit the available multiple orthogonal channels which is infeasible with a oneradio AP Mesh. A similar multi-radio architecture has been proposed in [8] where the channel used for any AP-AP link from among the available set is selected by sending probes to estimate the link round trip time (RTT) for the available channels and choosing the one with minimum RTT. Updating the channel allocation is performed periodically every few seconds. While the RTT is a useful indicator of channel load, it is a less-than-adequate metric for estimating interference due to simultaneous transmissions in a wireless scenario. Thus [8] protocol operates more as a loadbalancing scheme which improves but does not optimize aggregate network throughput.

Continuous monitoring of channel quality on all channels is infeasible with a single radio; two radios per node considerably simplifies this because this task can be performed by one radio while the other is transmitting data on the currently assigned channel. Suggestions for using one radio purely as a dedicated control channel and the other for data on all other channels have appeared for two-radio architectures [7] [6]; but these lead to low channel utilization due to control channel becoming a bottleneck, and offers no backward compatibility. Thus in our implementation, both radios are used to support data transmission. Nonetheless, irrespective of the specifics of how the two radios are used, this architecture allows the possibility of a fully distributed MAC implementation that is desirable for network robustness. For example, to eliminate the control channel bottleneck, we propose a new semidistributed AP-clustering approach. A distributed HighestConnectivity Cluster (HCC) algorithm [4] is employed to divide the network into AP clusters that are distinguished by the channel used for intra-cluster communication. Intercluster communication is performed using the (default and intra-cluster via the secondary) radio, respectively.

A common channel is used for all inter-cluster communications, and different channels are selected for intra-cluster communications by using a new Minimum Interference Channel Selection (MIX) algorithm. Control or management traffic uses only the default radio; while the secondary radio is only for data transmissions. Note that backward compatibility is achieved since this architecture allows a legacy single-radio AP to connect to the new two-radio APs through the (common) default radio.

Unlike most of other cluster-based networks (e.g. Bluetooth, UWB) that usually employ a cluster head as a

\footnotetext{
${ }^{1}$ More precisely, each AP is equipped with three radios, among which one is dedicated for tier-1 AP-MT (MT: Mobile Terminal) communications, and the other two are used for tier-2 AP-AP wireless mesh network. The need to prevent the interference between tier- 1 and tier- 2 networks promotes the use of dual, e.g. .11a $(5 \mathrm{GHz}) / \mathrm{b}(2.4 \mathrm{GHz})$, radio interface cards in such networks. In this paper, we focus on the performance of tier2 AP-AP network, and consider 802.11 b with three orthogonal channels in the simulation just for simplicity
}

controller running a centralized MAC, the architecture here uses clustering to only assign a channel in a distributed manner for the MAC; the base 802.11 MAC mechanisms are unchanged. Similar to [8], our protocol uses a virtual MAC address in place of the multiple physical MAC addresses used by two radios so that the higher (routing) layer sees only a single wireless network interface. Routing between the nodes is based on ad-hoc routing approaches similar to that in the traditional single-channel, single-radio mesh.

The rest of this paper is organized as follows: Section II discusses the key issues of maximizing both spatial reuse and channel reuse with multi-radio clustering. Section III presents the proposed multi-channel two-radio architecture with clustering. Section VI shows OPNET simulation results as well as the future work. Finally the paper is concluded in section $\mathrm{V}$.

\section{Maximizing both Spatial Reuse And Channel Reuse With Multi-Radio Clustering}

In [9] the authors investigated spatial reuse from a physical layer perspective. A homogeneous environment was assumed where every transmitter uses the same transmission power and data rate, and communicates to an immediate neighbor at the constant T-R distance $d$. Under such conditions, spatial reuse can be characterized by the distance between neighboring simultaneous transmitters (minimum T-T separation distance) that results in optimal spatial reuse. The authors investigated the optimal spatial reuse for two regular network topologies: the 1-D chain network and the 2-D grid network. Let $k$ denote such minimum T-T distance (also called spatial reuse factor), in number of hops (hop distance being $d$ ), then the lower bounds of $k$ for the two topologies are

$$
k= \begin{cases}{\left[2\left(1+\frac{1}{\gamma-1}\right) S_{0}\right]^{\frac{1}{\gamma}},} & \text { Chain network } \\ {\left[6\left(1+\frac{1}{\gamma-2}\right) S_{0}\right]^{\frac{1}{\gamma}},} & \text { 2-D hexogon }\end{cases}
$$

where $S_{0}$ denotes the Signal-Noise-Interference Ratio (SNIR) threshold, i.e., the minimum SNIR for achieving the maximum link throughput with a given raw data rate.

If we assume a perfect MAC protocol that schedules simultaneous communications only on transmitters that are $k$ hops away from each other, the network will be able to accommodate the maximum number of simultaneous transmitters, hence reaching its aggregate throughput limit. Hence the lower bounds of $k$ can be used to extrapolate to aggregate throughput limits. For example, in a chain network of $N$ nodes, where a packet will require relay by each of the $N-2$ intermediate nodes in order to be routed end-to-end, at most $N / k$ simultaneous transmitters can be supported in the chain. Let $C_{t h}$ denote the end-toend throughput, then

$$
C_{t h} \approx \frac{W}{k}
$$

where $W$ denotes the effective MAC layer data rate achieved at each relay, i.e. link capacity. 
Let $M$ denote the number of nodes for each cluster ${ }^{2}$ and $L$ denote the number of nodes every $k$ hops, then we have

$$
\begin{gathered}
M= \begin{cases}3, & \text { Chain network } \\
7, & \text { 2-D hexagon }\end{cases} \\
L= \begin{cases}k, & \text { Chain network } \\
(k+1) k+1, & \text { 2-D hexa gon }\end{cases}
\end{gathered}
$$

The detailed derivation of $L$ can be found in Appendix.

Obviously, $L / M$ gives the maximum number of different channels within $k$ hops. Intuitively, if the co-channel stations are separated more than $k$ hops, we will loose some spatial reuse. In order to incorporate more channels in the same area, a simple approach is to use multiple radios, which will allow a cluster working at multiple channels simultaneously. Given $N$ available channels and $R$ available radios per node, we have

$$
L / M \times R \geq N,
$$

which is the necessary condition for achieving maximum spatial reuse as well as taking advantages of all available orthogonal channels. Eq.5 can be simplified into

$$
R \geq \begin{cases}\frac{3}{k} N, & \text { Chain network } \\ \frac{7}{(k+1) k+1} N, & \text { 2-D hexagon }\end{cases}
$$

Denote the lower bound of $R$ as $R_{\min }$, leading to

$$
R_{\min }= \begin{cases}\left\lceil\frac{3}{k} N\right\rceil, & \text { Chain network } \\ \left\lceil\frac{7}{(k+1) k+1} N\right\rceil, & \text { 2-D hexagon }\end{cases}
$$

Table I shows the results of $R_{\min }$ for $802.11 \mathrm{~b}$ four data rates in chain topology and as well hexagon topology, where $W$ and $S_{0}$ were obtained using OPNET simulation with packet size 1000 bytes and $k$ was calculated with Eq.1. Notice that $k$ will enlarge as the network size decreases due to reduced interference. The results shown in Table I here are for an infinite topology, and therefore can be regarded as the lower-bound of $k$ and the upper-bound of $R_{\min }$ for limited-size networks. It is clear to see that one radio per cluster is enough to achieve maximum performance in a 2-D hexagon network using $802.11 \mathrm{~b}$.

\begin{tabular}{|c|c|c|c|c|}
\hline Data Rate (Mbps) & 1 & 2 & 5.5 & 11 \\
\hline$W$ (Mbps) & 0.89 & 1.5 & 3.5 & 5.0 \\
$S_{0}(\mathrm{~dB})$ & 11 & 14 & 18 & 21 \\
\hline$k$ (chain) & 3.4 & 4.2 & 5.7 & 7.2 \\
$R_{\min }$ (chain) & 3 & 3 & 2 & 2 \\
\hline$k$ (hexagon) & 5.3 & 6.7 & 9.1 & 11.5 \\
$R_{\min }$ (hexagon) & 1 & 1 & 1 & 1 \\
\hline
\end{tabular}

TABLE I

ONE-HOP PERFORMANCE OF 802.11B MAC WITHOUT RTS/CTS (OBTAINED FROM OPNET SIMULATION, $(\gamma=3, N=3)$ )

Next, we will propose a two-radio clustering architecture, where one (default) radio is used for all inter-cluster communications on a common channel, and the other (secondary) radio for communications among nodes within a cluster.

\footnotetext{
${ }^{2} \mathrm{Here}$, we assume HCC [4] algorithm is used to form clusters so that all cluster members are only one-hop away from their cluster-head.
}

\section{A Multi-Channel Two-Radio Architecture WITH Clustering}

A multi-channel architecture with clustering was previously studied in [5], which only considered a centralized TDMA MAC and one radio. Here, we propose to integrate two 802.11 radios (default and secondary) per node: the default radio is used for inter-cluster communications; while the secondary radio is for intra-cluster communications. Unlike most existing multi-channel approaches, the new clustered multi-channel two-radio (CMT) architecture not only eliminates the need for switching channels on a packetby-packet basis, but is also fully compatible with legacy devices. Fig.2 shows an example of a mesh network using the CMT architecture with three orthogonal channels, where each circle represents an independent cluster.

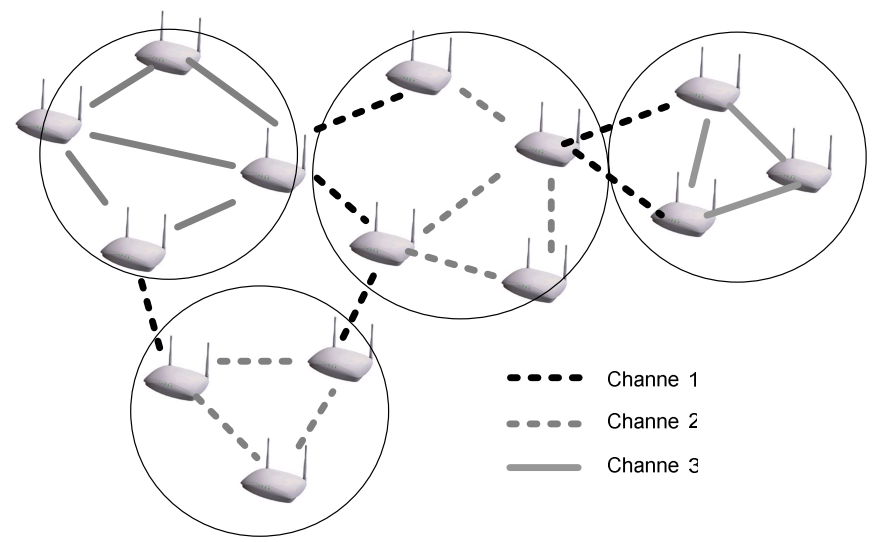

Fig. 2. Clustering Multiple Channels Architecture with Two Radios

Fig. 3 shows protocol stack in a two-radio device. We highlight the two new modules - MAC Extension and Secondary MAC/PHY - needed to enable the two-radio functionality. Algorithms in the new architecture are implemented in the MAC Extension. The secondary MAC/PHY has no administrative functionality, such as association, authentication etc. and can transmit only data traffic.

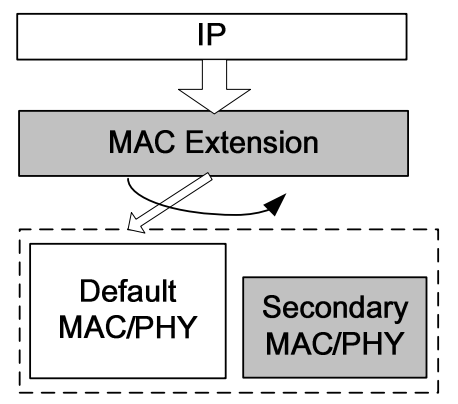

Fig. 3. Two-Radio Protocol Stacks

Clustering is accomplished by using the HighestConnectivity Cluster (HCC) algorithm first proposed in [4], based on the following rules:

- A node is elected as a clusterhead if it is the most highly connected (having the highest number of neighboring nodes) node of all its "uncovered" neighbor 
nodes (in case of a tie, lowest ID (e.g. MAC address) prevails);

- A node which has not elected its clusterhead is an "uncovered" node, otherwise it is a "covered" node;

- A node which has already elected another node as its clusterhead gives up its role as a clusterhead.

To minimize the co-channel interference (CCI) among clusters, we propose a Minimum Interference Channel Selection (MIX) algorithm, by which a clusterhead selects the secondary radio channel (denoted as $k$ ) with the minimum energy on air for intra-cluster communication.

Let $\bar{E}_{i j}$ denote the average energy on the $i$ th channel sensed by node $j$ for the duration $T$, we have

$$
\bar{E}_{i j}=\frac{\int_{t=t_{0}}^{t_{0}+T} E_{i j}(t) d t}{T},
$$

where $E_{i j}(t)$ is the instantaneous energy on the $i$ th channel at time $t$. Hence, the MIX algorithm is represented by

$$
\left\{k \mid \bar{E}_{k j}=\min \left(\bar{E}_{i j} \mid i=\{1,2, \ldots, n\}\right)\right\},
$$

where $n$ is the total number of orthogonal channels. Obviously, the longer the estimation duration $T$, the more accurate the estimation. Our simulations used $T=2$ seconds.

A clusterhead will generate a pseudo random number with 6 bits length for the ID of its cluster. Also it is responsible for notifying all its members which channel is used to configure the secondary radio as well as when the channel information is expired (denoted as $T_{E}$ (Eq.10)).

$$
T_{E}=T_{o}+T_{1}+\text { uniform }\left(0, T_{2}\right),
$$

where $T_{o}$ indicates the time when the clusterhead selected the channel, and $T_{1}$ and uniform $\left(0, T_{2}\right)^{3}$ give the constant and random components of the lifetime, respectively. Our simulations used $T_{1}=T_{2}=100$ seconds.

When the channel information is expired, the clusterhead will re-run the MIX algorithm to select a new channel, then broadcast the updated channel and its lifetime to its cluster members.

After getting the channel information, the neighboring nodes notify each other the channel used by their secondary radio. Thereby, we build a new 16-bit CMT field (see Fig.4) with three sub-fields: status, channel, and number of uncovered neighboring nodes. The "cluster-ID" flag indicates the cluster that the node belongs to, and is only meaningful when "status" is not "uncovered"; the "number of uncovered neighboring nodes" is used for electing clusterhead. In our OPNET implementation, the new 16-bit CMT field is added into the 802.11 DATA frame. The 16-bit "Duration ID" field in the legacy 802.11 ACK frame can also be used as the new CMT field, since it is meaningless when segmentation is not used or the ACK is for the last fragment of the packet.

After learning that a peer node belongs to the same cluster, a node will configure the forwarding table in its extended MAC such that all packets destined to the peer

\footnotetext{
${ }^{3}$ a random variable with uniform distribution on the range $\left(0, T_{2}\right)$; the random component is designed to avoid the event that two clusters always select the channel at the same time, i.e., channel selection collisions.
}

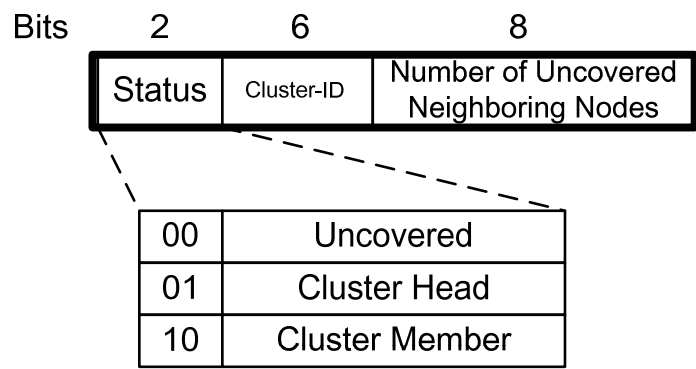

Fig. 4. Definition of 16-Bit CMT Field

node go through the secondary MAC/PHY module. Fig.5 summarizes the above clustering and channel selecting procedures with a state transition diagram.

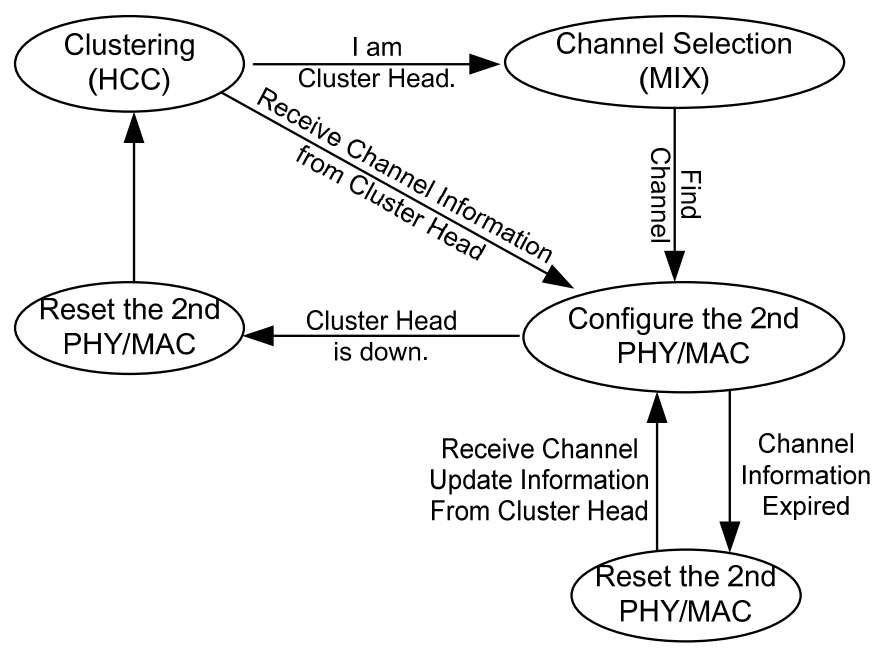

Fig. 5. A State Transition Diagram of Clustering and Channel Selecting Procedures

\section{Simulation Results AND Discussions}

Fig. 6 shows an example of how our clustering multichannel and two-radio architecture works in a $10 \times 10$ regular grid with three orthogonal channels. We implement a two-radio 802.11 client module in OPNET. Let $d$ denote the distance between two nearest neighbors; we configure the transmission range as $\sqrt{2} d$. During the simulation, the network will automatically cluster into the topology as shown in Fig.6. The dark nodes are clusterhead, and the dotted circle indicates all independent clusters. Two orthogonal channels (for the circles denoted by thick and thin dash lines, respectively) are used for intra-cluster communications. The channel assignment in Fig.6 minimizes co-channel interference and achieves the highest spatial reuse.

Fig.7 compares the total one-hop throughput with the new clustering multi-channel and two-radio architecture to the traditional single-channel, single-radio mesh. A random traffic generation model at each node was used with a sufficiently high offered load such that the nodes remain saturated during the simulation. Fig.7 clearly demonstrates the performance improvement with clustering and multiple 


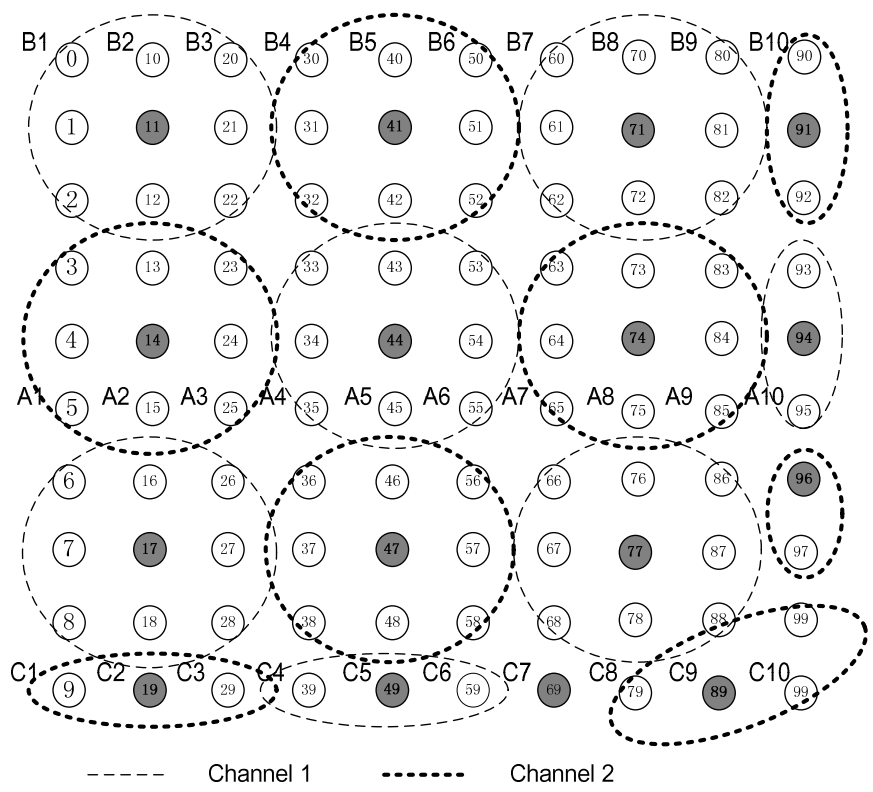

Fig. 6. An Example of $10 \times 10$ Grid Using the Clustering Multi-Channel and Two-Radio Architecture

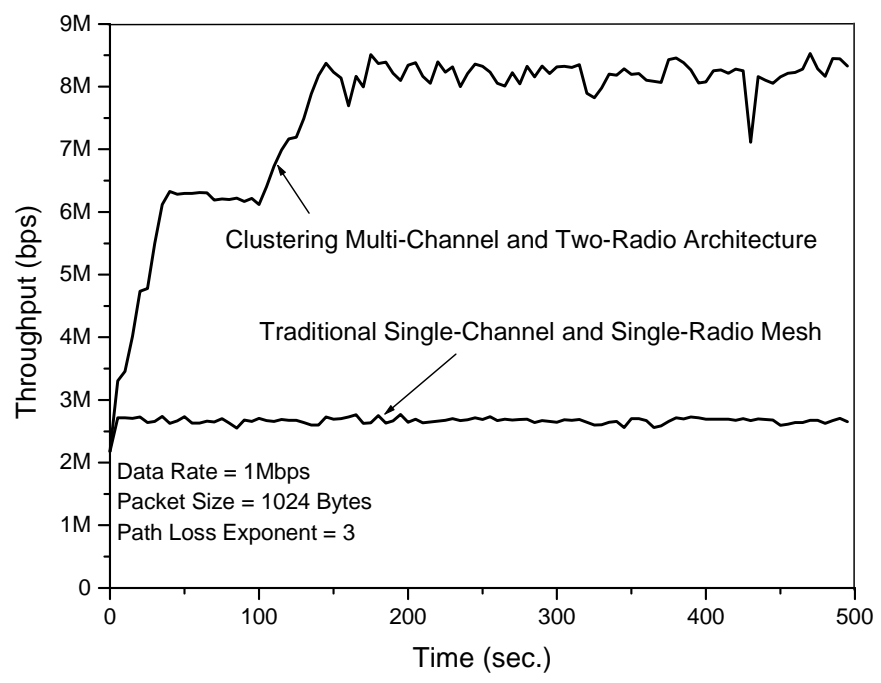

Fig. 7. Total One-Hop Throughput Comparison

orthogonal channels. The steady-state average throughput is 8.1 Mbps in the new CMT architecture, and only 2.7 Mbps in the single-channel and single-radio mesh. The gain is about $300 \%$, which is the maximum gain achievable when using 3 orthogonal channels.

Fig.8 illustrates the one-hop throughput distribution with respect to links, where links $A_{i}, B_{i}$, and $C_{i}$ are also indicated in Fig. $6(i=\{1,2, \ldots, 10\})$. Clearly, links $A_{i}$ experience worse interference environment than links $B_{i}$ and $C_{i}$, leading to the oscillation of the throughput distribution, illustrating the location-dependent fairness problem. We do not consider the fairness problem further; it is interesting to speculate how physical carrier sensing may be used to mitigate the location-dependent fairness problem. It implies for instance that the preferable locations for gateways in a wireless mesh network may not be the center of the network.

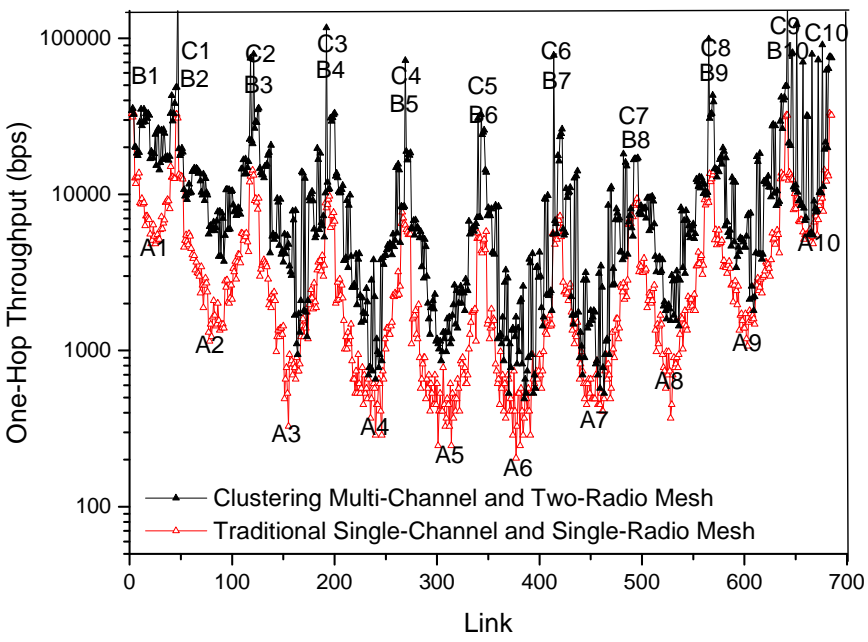

Fig. 8. Average One-Hop Throughput Distribution (between 300sec. and 500 sec., $N=3$ )

Finally, we validate the new architecture in a random topology as shown in Fig.9. The transmission range is fixed at 25 meters. We color-coded the graph in Fig.9. Red and blue indicates the nodes using channel 1 and channel 2 for their secondary radio, respectively; while black nodes are the single-node clusters. We also used the circles to illustrate the clusters with clusterhead in the center. Fig.10 compares the performance with both aggregate throughput and throughput distribution. We clearly see that (after 300 seconds) the aggregate throughput of the proposed architecture with $N=3$ (10Mbps) is almost 3 times higher than that of the traditional one (3.5Mbps).

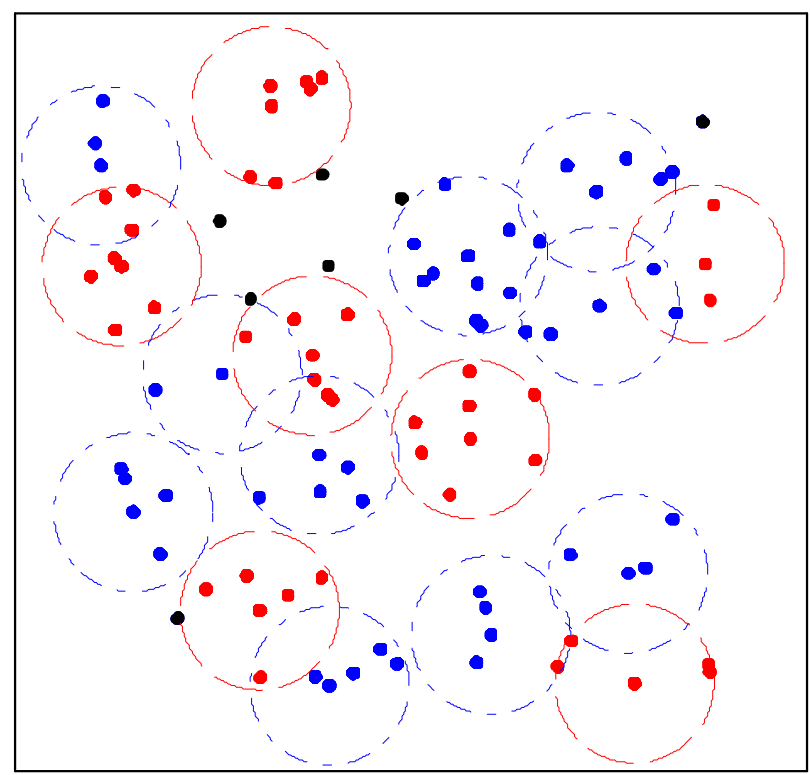

Fig. 9. 2-D 200m x 200m 100-Nodes Random Topology Using the Clustering Multi-Channel and Two-Radio Architecture (at 500 seconds, $N=3$ )

As we have mentioned in the introduction, spatial reuse and channel reuse are the key for high performance mesh. In the proposed two-radio architecture, the spatial reuse capability, however, will decrease as the number of available or- 


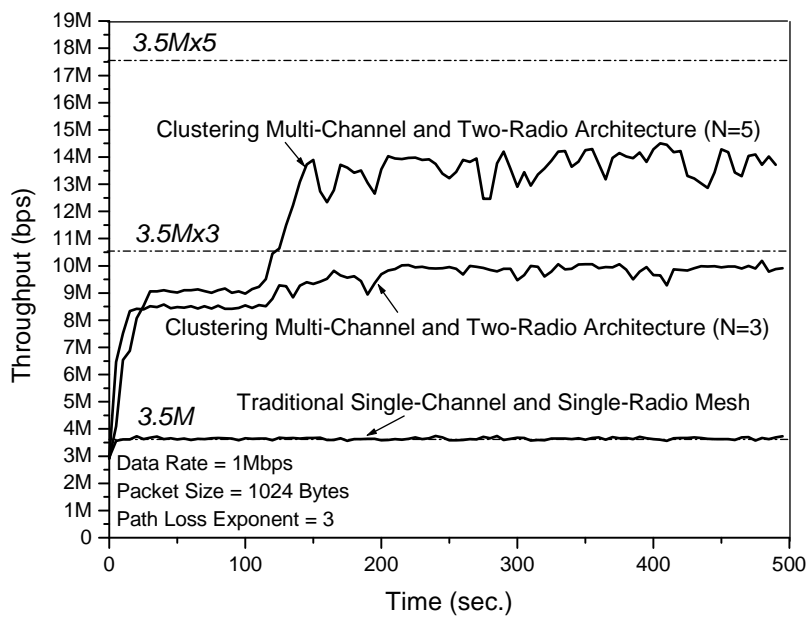

a) Tracing Aggregate Throughput

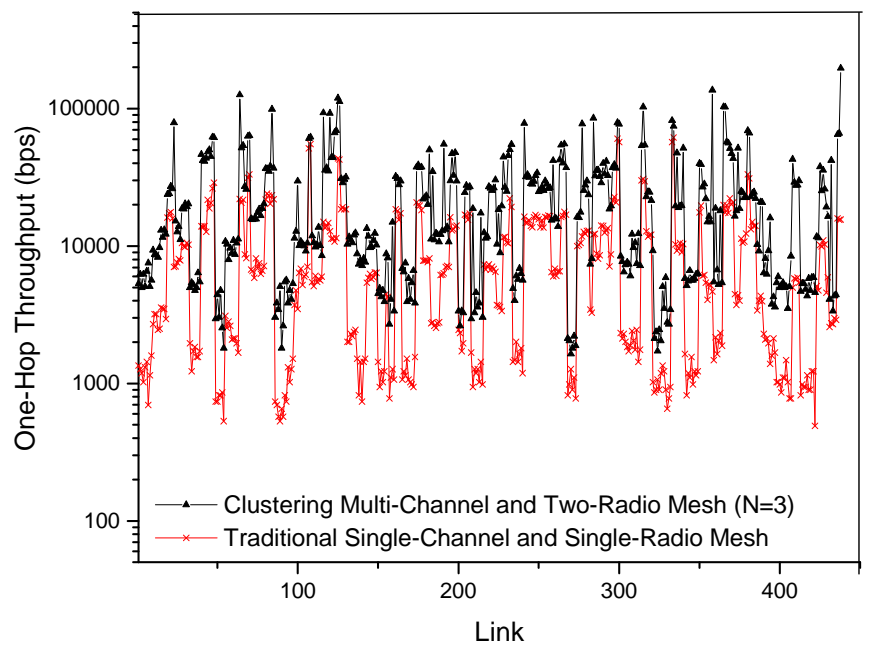

b) Throughput Distribution

Fig. 10. Performance Comparison in the Random Topology

thogonal channels increases due to the reduced per-channel network size. Fig.10a) also shows the effect of increasing channel number. Clearly, the performance with 5 orthogonal channels is far below the optimum $(=3.5 \mathrm{Mbps} \times 5$, where $3.5 \mathrm{Mbps}$ is the aggregate MAC throughput achieved for the same network with single channel). In order to maintain the same spatial reuse capability while increasing the number of orthogonal channel, we need to extend the current two-radio architecture to a multi-radio architecture, i.e., the number of radios per node $>2$. By allowing each cluster working on multiple channels simultaneously, i.e. multiple secondary radios, we can increase the per-channel network size so as to improve the spatial reuse. For example, the spatial reuse capability with 3 radios and 5 channels is the same as that with 2 radios and 3 channels 4

We also notice that the way of using a dedicated chan-

\footnotetext{
${ }^{4}$ The number of channels per secondary radio is fixed as 2 for both cases, excluding one radio and one channel dedicated for inter-clustering communications
}

nel for inter-clustering communications might become the bottleneck for the performance to continuously improve with the orthogonal channels increasing. One of our current works is to solve this problem by introducing multiple default radios. As shown in Fig.11, we have three neighboring clusters, all working on different channels. In order to allow them connect to each other, we can equip the edge nodes A, $\mathrm{B}$, and $\mathrm{C}$ with two default radios. Let's assume that cluster 1,2 , and 3 are using channel 1,2 , and 3 for their intraclustering communications, respectively. For node A with two default radios, the first one is configured with channel 2 , and the second one is configured with channel 3 , which will allow node A to connect to node B through the first default radio, and to node $\mathrm{C}$ through the second default radio. Clearly, the number of default radios is determined by the number of neighboring clusters.

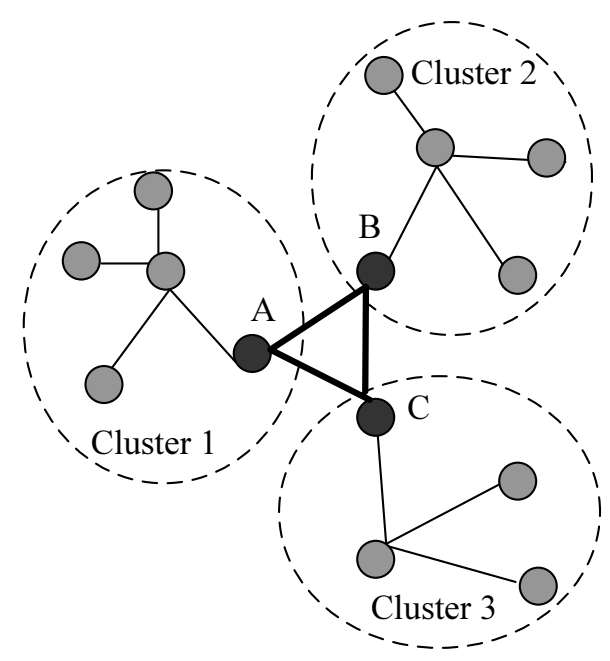

Fig. 11. Bridging in A Multi-Channel Clustering Network

All in all, the proposed two-radio architecture can be further extended to a multi-radio architecture, which is the key task of our future work.

\section{CONCLUSION}

In this paper, we have presented a two-radio architecture for an 802.11 AP mesh. Distributed clustering in conjunction with a new minimum interference channel selection algorithm (MIX) is used to distribute orthogonal channels in a mesh, thereby maximizing the aggregate throughput. OPNET simulations were conducted to validate performance; compared to a traditional single-channel and single-radio mesh, the gain achieved with three orthogonal channels in terms of the aggregate one-hop throughput is about $300 \%$ in a large scale grid.

\section{REFERENCES}

[1] MeshDynamics, http://www.meshdynamics.com.

[2] MeshNetworks, http://www.meshnetworks.com.

[3] Packethop, http://www.packethop.com 
[4] M. Gerla and J.T.-C. Tsai, "Multicluster, mobile, multimedia radio network", ACM/Baltzer Journal of Wireless Networks. vol. 1, (no. 3), 1995 , p. $255-265$.

[5] C. R. Lin, M. Gerla, "Adaptive Clustering for Mobile Wireless Networks," IEEE Jour. Selected Areas in Communications, pp. 12651275, Sept. 1997.

[6] N. Jain and S. Das, A Mutlichannel CSMA MAC Protocol with Receiver-Based Channel Selection for Mutlihop Wireless Networks, in Proceedings of the 9th Int. Conf. on Computer Communications and Networks (IC3N), Oct. 2001.

[7] S.-L. Wu, C.-Y. Lin, Y.-C. Tseng and J.-P. Sheu, A New MutliChannel MAC Protocol with On-Demand Channel Assignment for Multi-Hop Mobile Ad Hoc Networks, in IEEE Wireless Communications and Networking Conference (WCNC), Chicago, IL, Sept. 2000.

[8] A. Adya, P. Bahl, J. Padhye, A. Wolman, and L. Zhu, A Multi-Radio Unification Protocol for IEEE 802.11 Wireless Networks, Microsoft Research, Technical Report MSR-TR-2003-44, July, 2003.

[9] X. Guo, S. Roy, W. Steven Conner, Spatial Reuse in Wireless Ad-Hoc Networks, VTC2003.

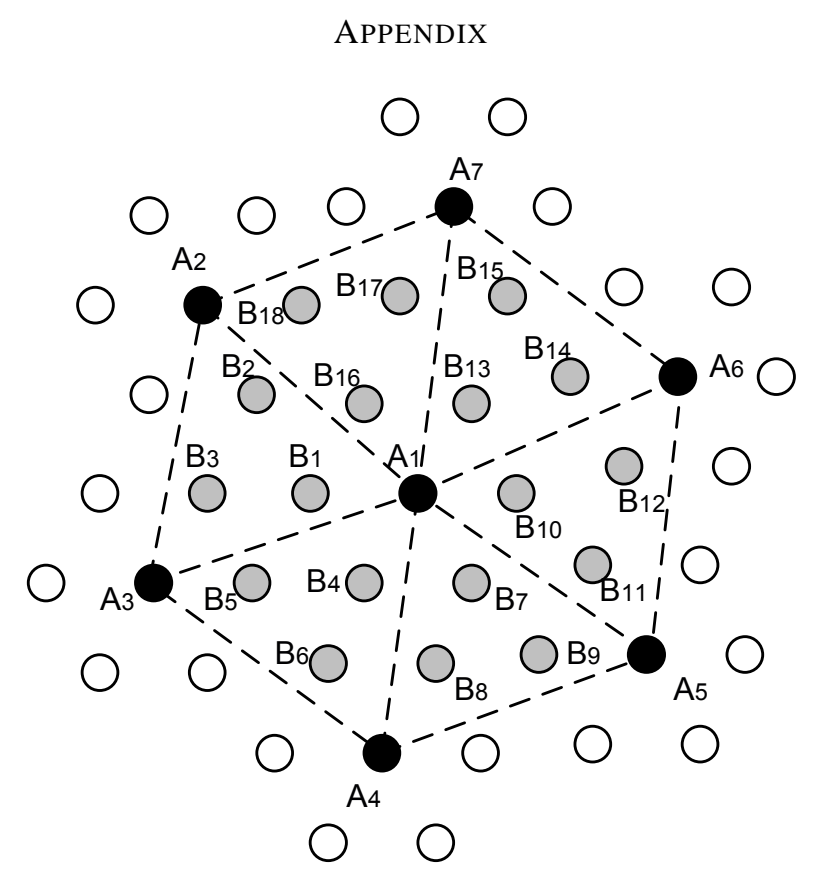

Fig. 12. 2D-hexagon Network

Fig.12 shows one part of an infinite hexagon network, where nodes $A_{i}(\mathrm{i}=\{1 \sim 7\}$ ) can transmit to one of its nearest neighbors simultaneously. In other words, those nodes are $k$ hops away to each other. Define all nodes within a hexagon with radius $k$ hops as a group. For example, nodes $B_{j}$ $(\mathrm{j}=\{1 \sim 18\})$ in Fig.12 are in the same group as node $A_{1}$. The total number of nodes in a group can be calculated as $\sum_{i=0}^{k}(6 i)$. However, such division leads to overlapping, i.e., each node belong to three groups. For example, nodes $B_{1}, B_{2}$, and $B_{3}$ are in the same group as node $A_{1}, A_{2}$, and $A_{3}$, respectively. Since the number of nodes every $k$ hops is given by the size of non-overlapped group, we can calculate it as:

$$
L=1+\frac{1}{3} \sum_{i=0}^{k}(6 i)=1+(k+1) k .
$$

For an infinite chain network, $\mathrm{L}$ is simply given by $k$. 